\title{
Reduction/Oxidation Induced Cleavable/Crosslinkable Temperature-Sensitive Hydrogel Network Containing Disulfide Linkages
}

\author{
Haeshin LeE and Tae Gwan PARK ${ }^{\dagger}$ \\ Department of Biological Sciences, Korea Advanced Institute of Science and Technology, \\ Yusong-gu Kusong-dong 373-1, Taejon 305-701, South Korea
}

(Received May 18, 1998)

\begin{abstract}
Temperature-sensitive poly( $N$-isopropylacrylamide) [poly(NIPAAm)] was crosslinked with a small amount of $N, N^{\prime}$-cystamine-bis-acrylamide which contains a disulfide linkage between two vinyl groups. The hydrogel network could be dissolved into water soluble polymer chains by the reduction of a disulfide bond to two thiol groups. The cleavage of crosslink points led to the experimental determination of the polymer molecular weight $\left(M_{n}\right)$ and the amount of free thiol concentration in the polymer backbone. This permitted the calculation of an average molecular weight between crosslinks $\left(M_{c}\right)$ which has been theoretically predicted by a Flory-Rehner equation based on either swelling or tensile experiments. The experimentally measured $M_{\mathrm{c}}$ values agree well with those obtained from the swelling experiment. When the reduced poly(NIPAAm) was re-oxidized in a dilute aqueous solution, time dependent evolution of inter- and intra-molecularly disulfide linked poly(NIPAAm) species could be observed.
\end{abstract}

KEY WORDS Poly( $N$-isopropylacrylamide) / Disulfide / Hydrogel / Lower Critical Solution Temperature / Reversible Gels /

Hydrogels are crosslinked polymer chain networks that swell in aqueous or biological solution. The structure of three dimensional polymer network has been mostly characterized from its equilibrium swelling capacity in aqueous water milieu. ${ }^{1}$ The degree of swelling for nonionic polymer hydrogels is determined by counter-balancing of mixing and elastic free energies that act in an opposite direction. ${ }^{2}$ The most critical factor affecting the swelling property is the crosslinking density that has been more conveniently defined as an average molecular weight between crosslinks $\left(M_{\mathrm{c}}\right)$. From the classical model of rubber elasticity, a Flory-Rehner equation was first introduced to estimate the $M_{\mathrm{c}}$, and later, a modified Bray-Merrill equation was developed. $^{3,4}$ The latter equation applies for crosslinking reactions of polymer in solid and solution states as well as the polymerization and subsequent network formation of various hydrophilic vinyl monomers in the presence of a small amount of crosslinker. The above equations were used to evaluate the theoretical calculation of $M_{\mathrm{c}}$ by simply measuring the equilibrium swelling capacity of hydrogel network. Crosslinking of polymer chains such as poly(vinyl alcohol) by radiation or chemical means easily permits the calculation of $M_{\mathrm{c}}$ because $M_{n}$ value of the polymer is known. ${ }^{5}$ However, when vinyl monomers are used for the network formation, $M_{n}$ value is not available. This problem limited the use of the above equation for free radically polymerized hydrogels based on vinyl monomers such as acrylamide and 2-hydroxyethylmethacrylate. ${ }^{6}$

Water soluble poly( $N$-isopropylacrylamide) [poly(NIPAAm)] has been extensively studied for its unique coil to globular conformation change with increasing the temperature above its lower critical solution temperature (LCST).$^{7-10}$ Crosslinked poly(NIPAAm) network that exhibits a volume phase transition at the LCST has also received much attention for its reversible swelling and deswelling properties useful for various novel applications. ${ }^{11}$ So far, poly(NIPAAm) hydrogels have been synthesized via a free radical polymerization by using a divinyl crosslinker like $N, N^{\prime}$-methylene-bis-acrylamide.

In this study, poly(NIPAAm) was crosslinked by using $N, N^{\prime}$-cystamine-bis-acrylamide (CBA) that contains a cleavable disulfide $-\mathrm{S}-\mathrm{S}$ - linkage in the structure. The disulfide bond in the hydrogel is readily cleaved by treating various reducing agents, resulting in a complete dissolution of the network structure into water soluble poly(NIPAAm) polymer chains. This makes it possible for the measurement of $M_{n}$, leading to the subsequent determination of $M_{\mathrm{c}}$ by assaying the amount of cleaved free thiol groups per poly(NIPAAm) chain. The experimentally obtained $M_{\mathrm{c}}$ value was compared with that calculated from using the Bray-Merrill equation based on a swelling experiment. In addition, the cleaved poly(NIPAAm) chains were re-oxidized to regenerate inter- and intra-molecularly disulfide linked poly(NIPAAm) aggregates, which were kinetically observed by an aqueous phase size exclusion chromatography.

\section{MATERIALS AND METHODS}

\section{Materials}

NIPAAm purchased from Eastman Kodak was recrystallized with hexane. CBA from Polysciences Co. was recrystallized with acetone. Ammonium persulfate (APS), $N, N, N^{\prime}, N^{\prime}$-tetramethylethylenediamine (TEMED), dithiothreitol (DTT), 5,5'-dithiobis(2-nitrobenzoic acid) [DTNB, Ellman's reagent], L-cysteine, and cystamine $\mathrm{HCl}$ were purchased from Sigma Co. All other chemicals were analytical grade.

\section{Methods}

Synthesis of Poly(NIPAAm) Gels Crosslinked with $C B A$. Two poly(NIPAAm) gels having different weight

\footnotetext{
† To whom all correspondence should be addressed (Tel: +82-42-869-2621, Fax: +82-42-869-2610, e-mail: tgpark@ sorak.kaist.ac.kr).
} 
ratios of CBA to NIPAAm $(2.5$ and $5 \%(\mathrm{w} / \mathrm{w}))$ were synthesized. The two gels were denoted as CBA $2.5 \%$ and $5.0 \%$ gels, respectively. A mixture of $10 \mathrm{~g}$ NIPAAm and 250 or $500 \mathrm{mg}$ CBA was dissolved in $70 \mathrm{ml}$ deionized water and nitrogen bubbled to remove residual oxygen. The monomer solution mixed with $0.1 \mathrm{ml}$ of $10 \%(\mathrm{w} / \mathrm{v})$ APS and $0.1 \mathrm{ml}$ of TEMED was injected into a gelation mold consisting of the two glass plates separated by a $3 \mathrm{~mm}$ thick rubber spacer (Bio-Rad). The polymerization was carried out at room temperature for $5 \mathrm{~h}$. The gel sheet was washed with deionized water and dried under vacuum.

Characterization of Poly(NIPAAm) Gels. Equilibrium swelling capacities of two poly(NIPAAm) gels were determined gravimetrically by equilibrating dried polymer gel sheets in deionized water at $25^{\circ} \mathrm{C}$. Wet weight and dry weights were used for the calculation. Swelling capacity values of the gels at a relaxed state were obtained from the measurement of gel volume right after crosslinking. The density of dry poly(NIPAAm) gel was obtained from a volume replacement method using a non-swelling solvent, mineral oil.

Reductive Dissolution of Poly(NIPAAm) Gels by DTT. Dried poly(NIPAAm) gel sheet $(1-1.5 \mathrm{~g})$ was chopped and placed in $400 \mathrm{ml}$ of $0.1 \%(\mathrm{w} / \mathrm{v}) \mathrm{DTT} / 0.1 \mathrm{M}$ phosphate buffer solution ( $\mathrm{pH} 8$ ). The cleavage reaction of $-\mathrm{S}-\mathrm{S}$ - linkage in the gel was carried out for $40 \mathrm{~h}$ at room temperature under stirring condition. The digestion of the gel was performed under nitrogen atmosphere to prevent re-oxidation. The white colored solution containing many small gel particles and undissolved debris was centrifuged at $12000 \mathrm{rpm}$ for $15 \mathrm{~min}$, and filtered through a $0.45 \mu \mathrm{m}$ cellulose acetate filter. The clear solution was further purified by using an Amicon ultrafiltration membrane (molecular weight cutoff: 3000 ) under nitrogen pressure. During the ultrafiltration process, the phosphate buffer was changed to $0.1 \mathrm{M}$ citrate buffer $(\mathrm{pH} 4)$ to minimize re-oxidation reaction of thiol groups in the polymer backbone. The concentrated water soluble poly(NIPAAm) was freeze dried and stored at $-20^{\circ} \mathrm{C}$.

Characterization of Dissolved Poly(NIPAAm). The LCST of cleaved poly(NIPAAm) was determined at different $\mathrm{pH}$ values by measuring a transmittance change at $600 \mathrm{~nm}$ as a function of temperature. Poly(NIPAAm) solution with the concentration of $1 \mathrm{mg} \mathrm{ml}^{-1}$ was prepared with $5 \mathrm{mM}$ Tris buffer. The polymer solution was titrated to $\mathrm{pH} 3.8,6.0,7.0$, and 8.0. The amount of free thiol (sulfhydryl) groups present in the poly(NIPAAm) backbone was determined by using an Ellman's reagent which specifically reacts with thiols at neutral $\mathrm{pH} .{ }^{12}$ Briefly, $4 \mathrm{mg}$ Ellman's reagent dissolved in $1 \mathrm{ml}$ of $0.1 \mathrm{M}$ phosphate buffer ( $\mathrm{pH} \mathrm{8.0)}$ was prepared and $50 \mu$ lof this solution added with $2.5 \mathrm{ml}$ buffer solution was reacted with poly(NIPAAm) samples $\left(10 \mathrm{mg} \mathrm{ml}^{-1}\right)$ for $15 \mathrm{~min}$ at room temperature. The yellow color developed was monitored at $412 \mathrm{~nm}$. The concentration of thiols in the poly(NIPAAm) was then calculated by using L-cysteine as a calibration standard. Molecular weights of cleaved poly(NIPAAm) were determined by an aqueous phase gel filtration chromatography. Bio-silect $250-5$ column having $300 \times 7.8 \mathrm{~mm}$ dimension (Bio-Rad) was used for Waters $600 \mathrm{E}$ high performance

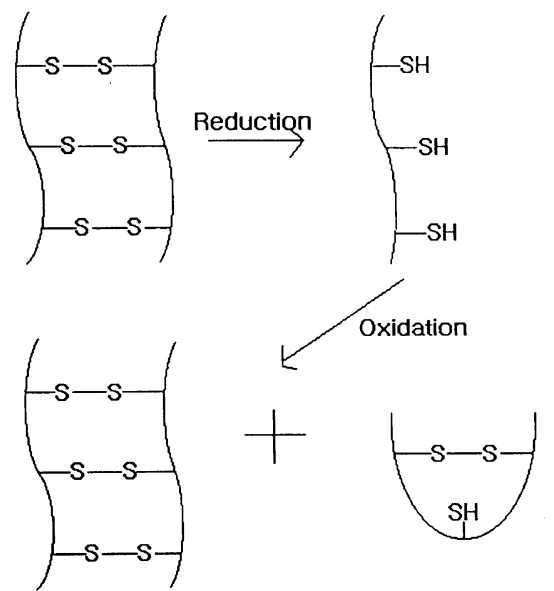

Figure 1. Schematic illustration of reduction/oxidation induced cleavage/crosslinking of poly(NIPAAm) gel containing disulfide bridges as crosslinks.

liquid chromatography (HPLC) system with $230 \mathrm{~mm}$ UV monitoring. As a mobile phase, $0.1 \mathrm{M}$ phosphate $/ 0.05 \mathrm{M}$ sodium chloride ( $\mathrm{pH}$ 6.0) was used and the sample injection volume was $20 \mu \mathrm{l}$. For the calibration of molecular weights, ovalbumin (44000), human growth hormone $\left(M_{w} 22000\right)$, equine myoglobin (17000), and hen egg white lysozyme (14300) were used.

Re-oxidation of poly(NIPAAm) containing free thiol groups. Cleaved poly(NIPAAm) containing free thiol groups was dissolved in $0.1 \mathrm{M}$ phosphate buffer $(\mathrm{pH} 8.0)$ at the concentration of $1 \mathrm{mg} \mathrm{ml}^{-1}$ in the presence and absence of an oxidation aiding agent, $0.2 \mathrm{M}$ cystamine $\mathrm{HCl}$, under an aeration condition. Alternatively, the re-oxidation reaction was also carried out by irradiating ultrasonic wave without using cystamine $\mathrm{HCl}$. The aerated polymer solution was placed in an ultrasonic bath (Branson) radiating a power of $47 \mathrm{kHz} \pm 6 \%$ and 120 watts. The sample was exposed to the ultrasonic wave with $10 \mathrm{~min}$-on and $5 \mathrm{~min}$-off cycles. The evolution of inter- and intra-molecularly re-shuffled poly(NIPAAm) species via a disulfide formation was identified as a function of time by the aqueous gel filtration chromatography. The formation of disulfide crosslinked poly(NIPAAm) species was also confirmed by observing the change in viscosity using a falling ball viscometer (Gilmont).

\section{RESULTS AND DISCUSSION}

The solubilizable hydrogels synthesized with the crosslinker containing a disulfide bond were first reported for the purpose of polyacrylamide gel electrophoresis that separates proteins and nucleic acids by an electric field. ${ }^{13}$ These gels can be dissolved by treating with reducing agents, providing facile extraction recovery of separated protein and DNA species out of a crosslinked gel slice. Polyoxazoline gels having disulfide bridges were synthesized for reversible inter-conversion of sol and gel states. ${ }^{14}$ Very recently, solubilized polyacrylamide chain containing thiol groups which was obtained from the reduction process was re-oxidized and crosslinked back for the entrapment of islets cells for re-fillable bioartificial pancreas. ${ }^{15}$ As shown in Figure 1, synthesized poly(NIPAAm) gel network could be 


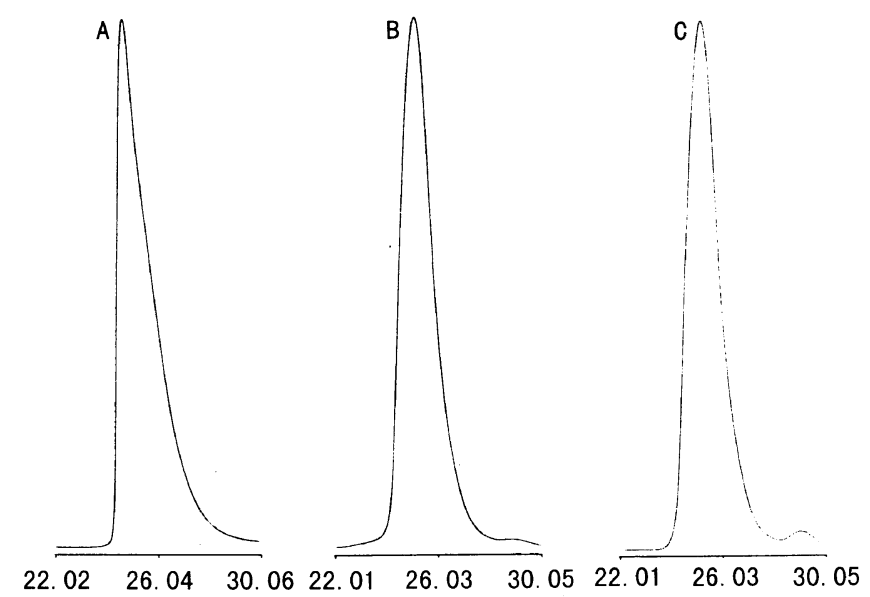

Figure 2. Aqueous GPC profiles of lysozyme (A), dissolved poly(NIPAAm) from $2.5 \%$ CBA gel (B), and dissolved poly(NIPAAm) from $2.5 \%$ CBA gel (C).

dissolved into water soluble polymer chains by treating with a reducing agent, DTT. However, the reductive cleavage reaction of disulfide linkage in the poly(NIPAAm) gel did not yield a complete dissolution of the gel. This was likely caused by the use of stoichiometrically unbalanced amount of DTT in the reduction process. The concentration of DTT, which intra-molecularly oxidizes itself, was critical for completely breaking up of -S-S- linkages in the gel. Alternatively, it can be conceived that the poly(NIPAAm) gel had an inhomogeneous structure composed of both densely and lightly crosslinked regions, and DTT preferably digested the lightly crosslinked region.

The molecular weight of recovered water soluble fraction was determined by an aqueous gel permeation chromatography (GPC). Number average molecular weights $\left(M_{n}\right)$ of the two water soluble poly(NIPAAm) chains obtained from different concentrations of CBA concentrations in the gel were very similar, 13300 for $2.5 \%$ CBA gel and 13200 for $5.0 \%$ CBA gel. These molecular weight values determined might be underestimated if the reductive cleavage reaction occurred in the lightly crosslinked region of the gel.

Surprisingly, both of the two poly(NIPAAm) have a near monodisperse molecular weight distribution as shown in Figure 2, which exhibits a sharp and narrow peak in the GPC chromatogram, similar to that of monodisperse protein standard, lysozyme $\left(M_{w} 14400\right)$. The monodisperse molecular weights of poly(NIPAAm) obtained from the network structure can be attributed to the opaque gelation phenomenon of poly(NIPAAm) during the polymerization. When the polymerization proceeded in a mold at room temperature, clear monomer NIPAAm solution gradually turned into a white and milky gel by undissipated local heat generated from the polymerization that raised the polymerization temperature above the LCST. As a result, growing polymer chains collapsed and precipitated out of solution. A reactive free radical present at one growing polymer chain end could be sterically hidden and terminated in the collapsed polymer chains due to a diffusion limited monomer accessibility. This might be the reason for the observed monodisperse molecular weight of poly(NIPAAm) in the gel. It appears that the molecular weight of poly-

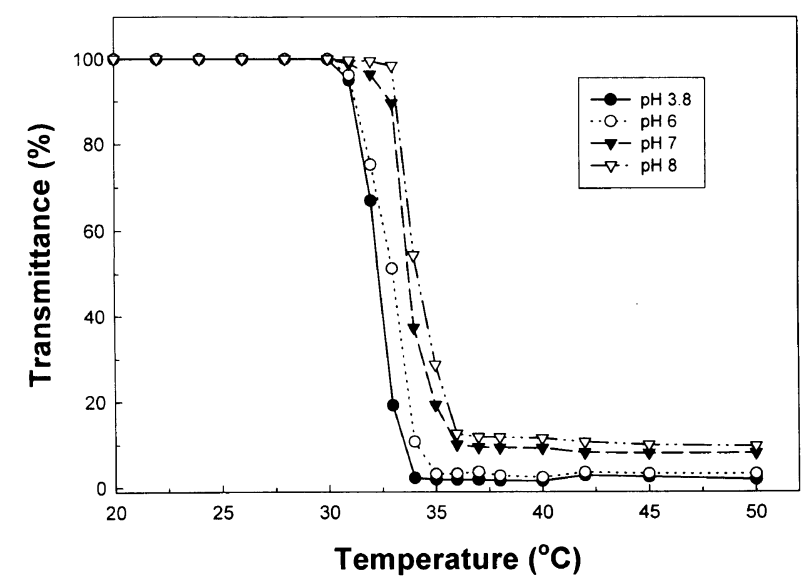

Figure 3. LCST of cleaved poly(NIPAAm) from $5 \%$ CBA gel as a function of $\mathrm{pH}$.

(NIPAAm), 13300, is near a critical growing polymer chain length required for the steric termination of free radical polymerization. The opaque poly(NIPAAm) gel did not go back reversibly to a transparent gel upon cooling below the LCST, suggesting a macroscopically heterogeneous gel structure in nature. ${ }^{16}$ It should be mentioned that the molecular weights of poly(NIPAAm) determined by the aqueous GPC method are relative values to a series of protein standards that are in a folded state in aqueous solution.

According to the result of the Ellman's assay used for a thiol group titration, average number of - $\mathrm{SH}$ group present in a single poly(NIPAAm) chain for $2.5 \%$ and $5.0 \%$ CBA gels were 2.9 and 5.0, respectively. On the basis of relative molar ratio of CBA to NIPAAm, the incorporation efficiency of feeding CBA crosslinker present in the initial polymerization recipe into the poly(NIPAAm) network formation was calculated. It turns out that $2.5 \%$ and $5.0 \%$ gels contained 5.2 and 4.5 fold higher relative molar ratios of CBA/NIPAAm, respectively, compared to those in initial crosslinker/ monomer feed molar composition. This suggests that a large fraction of homo-poly(NIPAAm) was generated during the gel network formation. The homo-polymer fraction was removed during the washing step of the gel sheet.

Figure 3 shows the LCST behaviors of the poly(NIPAAm) chains obtained from $5.0 \%$ CBA gel as a function of $\mathrm{pH}$. It can be seen that the LCST shifts to higher temperature (from 32 to $34^{\circ} \mathrm{C}$ ) with increasing the $\mathrm{pH}$ value, suggesting that a thiol, $-\mathrm{SH}$, group in the polymer chain is ionized and becomes a thiolate group. The small increase in the LCST while maintaining its sharpness, caused by the $\mathrm{pH}$-induced protonation-deprotonation of $-\mathrm{SH}$ group, can be understood by the fact that as hydrophilic or ionic co-monomer is incorporated into poly(NIPAAm) chain, the LCST shifts to higher temperature. ${ }^{17}$ Because the $\mathrm{p} K_{\mathrm{a}}$ value of $-\mathrm{SH}$ depends on the hydrophilic/hydrophobic microenvironment, as reported in different $\mathrm{p} K_{\mathrm{a}}$ values of cysteine $-\mathrm{SH}$ in the case of protein, ${ }^{18}$ it can be deduced from Figure 3 that $\mathrm{p} K_{\mathrm{a}}$ of $-\mathrm{SH}$ in the poly(NIPAAm) chain is located between $\mathrm{pH} 6$ and 7 .

From the data of $M_{n}$ and the number of $-\mathrm{SH}$ groups in the polymer chain, we can now determine $M_{\mathrm{c}}$ ex- 
perimentally. The determined $M_{\mathrm{c}}$ values were 4550 and 2650 for 2.5 and $5.0 \%$ CBA gels, respectively. The conventional method to determine $M_{\mathrm{c}}$ in the case of network formation by polymerization from vinyl monomers is based on the following equation, ${ }^{4}$ which is given below,

$$
\frac{1}{M_{\mathrm{c}}}=\frac{2}{M_{n}}-\frac{\left(\frac{v}{V_{1}}\right)\left[\ln \left(1-v_{2, \mathrm{~s}}\right)+v_{2, \mathrm{~s}}+\chi_{1} v_{2, \mathrm{~s}}^{2}\right]}{v_{2, \mathrm{r}}\left[\left(\frac{v_{2, \mathrm{~s}}}{v_{2, \mathrm{r}}}\right)^{1 / 3}-\frac{1}{2}\left(\frac{v_{2, \mathrm{~s}}}{v_{2, \mathrm{r}}}\right)\right]}
$$

where $M_{\mathrm{c}}$ is molecular weight between crosslinks in the gel, $M_{n}$ is number average molecular weight, $v$ is specific volume of polymers $\left(0.990 \mathrm{~cm}^{3} \mathrm{~g}^{-1}\right.$ and $0.952 \mathrm{~cm}^{3} \mathrm{~g}^{-1}$ for samples of 2.5 and $5.0 \%$ CBA to NIPAAm feed weight ratio, respectively), $V_{1}$ is solvent molar volume, $\chi_{1}$ is a Flory-Huggins interaction parameter of poly(NIPAAm) in water at $25^{\circ} \mathrm{C}(0.52$ as obtained from the literature $\left.{ }^{19}\right), v_{2, \mathrm{r}}$ and $v_{2, \mathrm{~s}}$ are defined below,

$$
v_{2, \mathrm{r}}=\frac{w_{\mathrm{p}}}{w_{\mathrm{p}}+\left(w_{\mathrm{r}}-w_{\mathrm{p}}\right)\left(\frac{\rho_{\mathrm{p}}}{\rho_{\mathrm{r}}}\right)} \quad v_{2, \mathrm{~s}}=\frac{w_{\mathrm{p}}}{w_{\mathrm{p}}+\left(w_{\mathrm{s}}-w_{\mathrm{p}}\right)\left(\frac{\rho_{\mathrm{p}}}{\rho_{\mathrm{s}}}\right)}
$$

where $w_{\mathrm{s}}$ is the weight of fully swollen gel, $w_{\mathrm{r}}$ is the weight of gel in a relaxed state, and $w_{\mathrm{p}}$ is the weight of dry gel; $\rho_{\mathrm{p}}$ and $\rho_{\mathrm{s}}$ are the densities of polymer and solvent, respectively. The parameters, $w_{\mathrm{s}}$ and $w_{\mathrm{r}}$ in the above equation were obtained from the swelling experiment of the gel in deionized water at $25^{\circ} \mathrm{C}$ and by measuring the weight of the gel right after crosslinking at $25^{\circ} \mathrm{C}$. The theoretical calculation of $M_{\mathrm{c}}$ yielded 5220 and 2590 for 2.5 and $5.0 \%$ CBA gels, respectively. These calculated $M_{\mathrm{c}}$ values were very comparable to those values of 4550 and 2650 which were experimentally determined, validating the above theoretical equation derived from a rubber elasticity based on classical thermodynamic considerations. However, it should be mentioned that the determination of absolute $M_{n}$ values of cleaved poly(NIPAAm) is highly desirable to strictly compare the difference between experimental and theoretical values. Additionally, it was assumed that the hydrogel prepared in the present study has a homogeneous microscopic structure in terms of crosslinking density, and the CBA crosslinker was $100 \%$ incorporated in the gel without generating pendant uncrosslinked vinyl groups in the polymer backbone. Although the theoretical equation is based on the homogeneous gel structure and CBA was not completely incorporated in the poly(NIPAAm) backbone in reality, the experimentally obtained $M_{\mathrm{c}}$ values are in good agreement with those calculated from the equation.

Since the water soluble poly(NIPAAm) chain contains free thiol groups, it is of particular interest to see the results of their re-oxidation, which would produce inter- and intra-molecular crosslinked poly(NIPAAm) species. This is a similar situation to a protein refolding process from an uncrosslinked and unfolded state, where a polypeptide chain having multiple cysteine residues is spontaneously re-shuffled to find a correct pathway to the most stable, energetically minimized conformation. ${ }^{20}$ In the case of poly(NIPAAm), because of its repeating

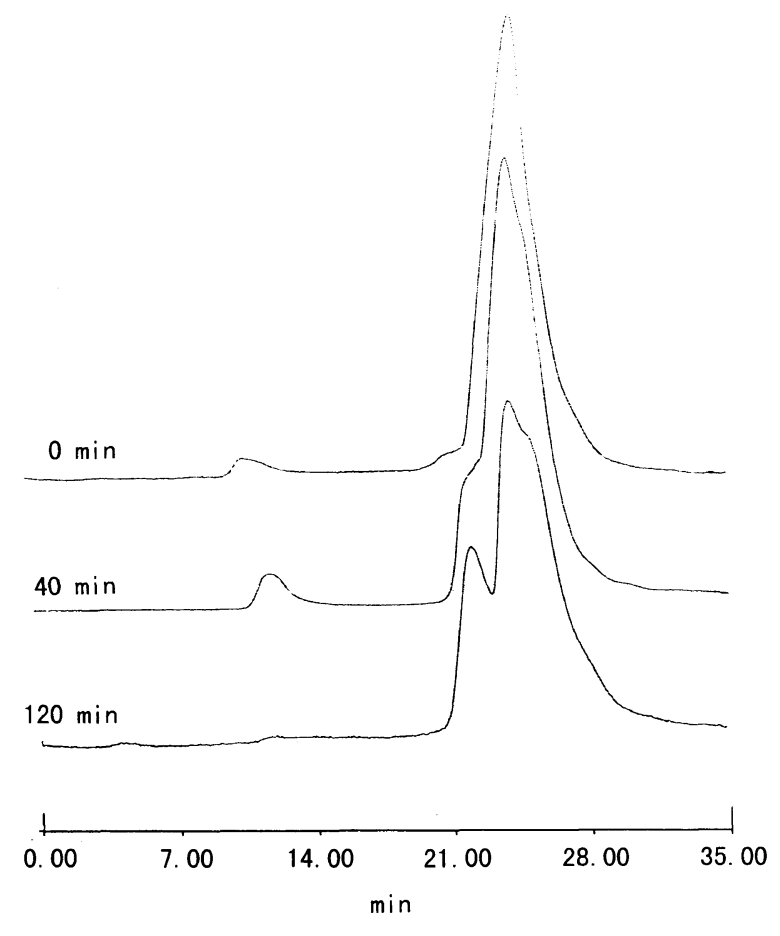

Figure 4. GPC profiles of disulfide crosslinked poly(NIPAAm) chains from $5 \%$ CBA gel by the re-oxidation process.

monomer structural units in the polymer backbone in contrast to polypeptides, it is likely that the re-shuffling process randomly occurs. Figure 4 shows GPC profiles during the re-oxidation process of dilute poly(NIPAAm) in aqueous solution with the aid of cystamine as an oxidation agent. It can be seen that an initial single peak of monomeric poly(NIPAAm) chain which elutes at $24.2 \mathrm{~min}$ splits into multiple three peaks appearing at $22.2,24.2$, and $25.5 \mathrm{~min}$ with time. The earlier peak at $22.2 \mathrm{~min}$ corresponds to a dimer of poly(NIPAAm) generated by inter-molecular di-sulfide formation, and the later eluting shoulder peak at $25.5 \mathrm{~min}$ is likely to be a monomeric folded poly(NIPAAm) having intramolecularly crosslinked di-sulfide linkages. The evolution of inter- and intra-molecularly $-\mathrm{S}-\mathrm{S}$ - crosslinked poly(NIPAAm) species can be regarded as the protein refolding process from an unfolded state, which often generates aggregated and mis-folded species besides a native conformation. ${ }^{21}$ Because dilute polymer concentration was used for the re-oxidation, only dimeric poly(NIPAAm) was obtained. The addition of cystamine that has a -S-S- bond in its structure was essential for the facile formation of re-shuffled and crosslinked poly(NIPAAm) chains, since it provided an appropriate reduction/oxidation potential in the medium for the thiol-thiol oxidation. This is shown in Figure 5, in which only poly(NIPAAm) solution in the presence of cystamine increases its viscosity with time due to the formation of crosslinked dimeric poly(NIPAAm) species.

The re-oxidation and subsequent disulfide crosslinking reaction of thiol containing poly(NIPAAm) chains could also be induced by an exposure to ultrasonication without using any oxidation agents. It has been known that the ultrasonic treatment to an oxygen dissolved aqueous solution generates various reactive hydroxyl, peroxide, and superoxide radicals. ${ }^{22}$ It was reported that non- 


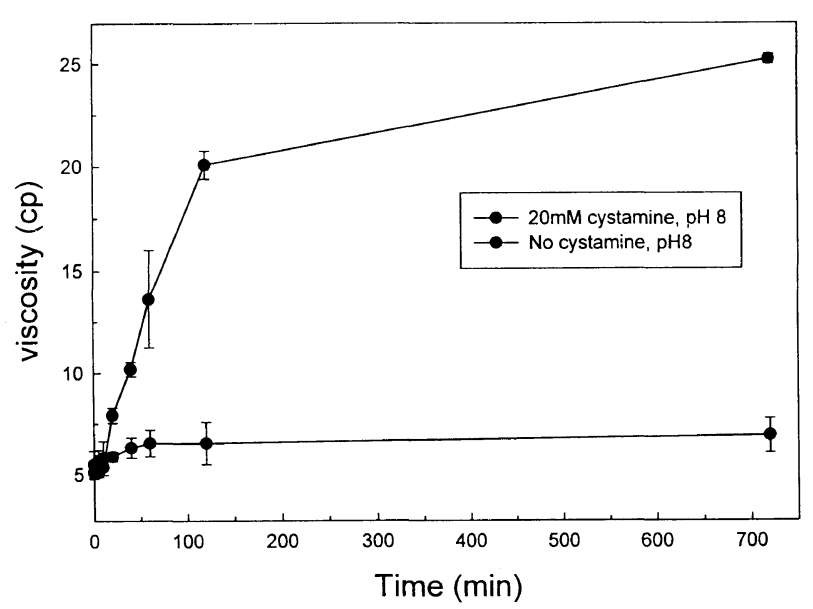

Figure 5. Viscosity change of poly(NIPAAm) chains during the re-oxidation process.

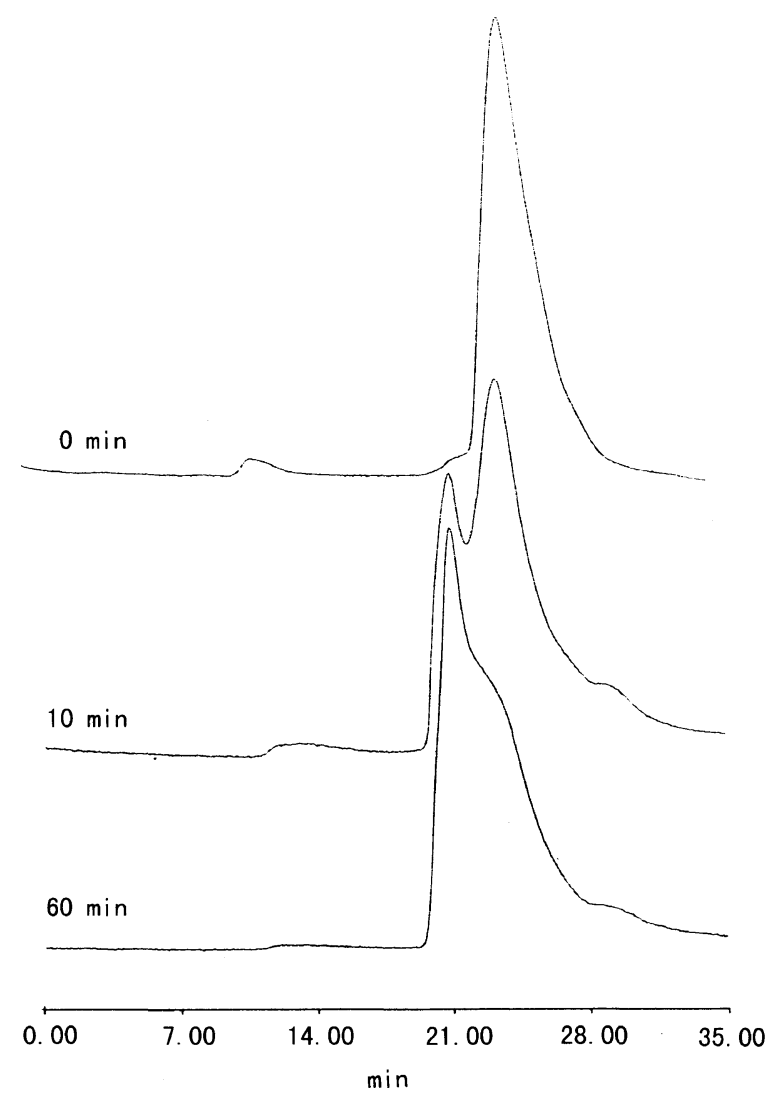

Figure 6. GPC profiles of ultrasonic irradiated poly(NIPAAm) chains as a function of time.

aqueous oil filled crosslinked protein capsules could be generated by the irradiation of ultrasonication to oilin-water droplets stabilized adsorbed bovine serum albumin at the interface. ${ }^{23}$ It was hypothesized that thiol groups in the poly(NIPAAm) chain could be oxidized by reactive radicals, and crosslinked themselves via $-\mathrm{S}-\mathrm{S}-$ linkages between the polymer chains. Figure 6 shows the GPC results of ultrasonically irradiated poly(NIPAAm) in the aqueous solution. It can be seen that an early eluting peak appearing at $20.9 \mathrm{~min}$, being multi-meric poly(NIPAAm) aggregates, grows gradually while the monomer peak diminishes concomitantly. It is also noted that there is no apparent intra-molecularly crosslinked form of poly(NIPAAm) which was eluted as a small shoulder peak in the GPC result obtained by using cystamine as an oxidant. This is attributed to the enhanced inter-molecular collision frequency between poly(NIPAAm) chains by the ultrasonic treatment. The GPC results also reveal that the re-oxidation yield, as judged from the relative peak ratio of multimer and monomer, was enhanced much greater by an ultrasonic treatment than by an chemical means, supporting the above postulation.

In conclusion, it has been demonstrated that poly(NIPAAm) was crosslinked by a crosslinker containing a disulfide bond and could be re-dissolved into water soluble poly(NIPAAm) chains by the cleavage of disulfide crosslinks. This permits the experimental determination of $M_{\mathrm{c}}$ for the first time, and the obtained $M_{\mathrm{c}}$ values were very similar to those of theoretically calculated ones. The reduced poly(NIPAAm) chains containing free thiol groups could be re-oxidized and subsequently inter- and intra-molecularly crosslinked poly(NIPAAm) could be regenerated. This study provides a good model system for simulating a protein refolding process.

Acknowledgment. This study was supported in part from the 1998 Biochemical Engineering Research Fund from the Korea Research Foundation, South Korea.

\section{REFERENCES}

1. N. A. Peppas and B. D. Barr-Howell, "Characterization of the Crosslinked Structure of Hydrogels," in Hydrogels in Medicine and Pharmacy, Vol. I, Fundamentals, N. Peppas, Ed., CRC Press, Boca Raton, FL, 1986, pp 27-56.

2. P. J. Flory, "Principles of Polymer Chemistry," Cornell University Press, Ithaca, N.Y., 1953, p 576.

3. P. J. Flory and R. J. Rehner, Chem. Phys., 11, 521 (1943).

4. J. C. Bray and E. W. Merrill, J. Appl. Polym. Sci., 17, 3779 (1973).

5. N. A. Peppas and R. E. Benner, Biomaterials, 1, 158 (1982).

6. N. A. Peppas, H. J. Moynihan, and L. M. Lucht, J. Biomed. Mater. Res., 19, 397 (1985).

7. M. Heskins and J. E. Guillet, J. Macromol. Sci. Chem., 2, 1441 (1969).

8. H. G. Schild, Prog. Polym. Sci., 17, 163 (1992).

9. E. I. Tiktopulo, V. E. Bychkova, J. Ricka, and O. B. Ptitsyn, Macromolecules, 27, 2879 (1994).

10. T. G. Park and A. S. Hoffman, Macromolecules, 26, 5045 (1993).

11. T. G. Park and A. S. Hoffman, Biotech. Bioeng., 35, 152 (1990).

12. G. L. Ellman, Arch. Biochem. Biophys., 82, 70 (1959).

13. J. N. Hansen, Anal. Biochem., 76, 37 (1980).

14. Y. Chujo, K. Sada, A. Naka, R. Nomura, and T. Saegusa, Macromolecules, 26, 883 (1993).

15. N. Hisano, N. Morikawa, H. Iwata, and Y. Ikada, J. Biomed. Mater. Res., 40, 115 (1998).

16. T. G. Park and A. S. Hoffman, J. Appl. Polym. Sci., 52, 85 (1994).

17. T. G. Park and A. S. Hoffman, J. Appl. Polym. Sci., 46, 659 (1992).

18. P. C. Jocelyn, "Biochemistry of the SH group," Academic Press, New York, N.Y., 1972.

19. Y. H. Bae, T. Okano, and S. W. Kim, J. Polym. Sci., Polym. Phys., 28, 923 (1990).

20. T. E. Creighton, J. Phys. Chem., 89, 2452 (1985).

21. T. E. Creighton, Biochem. J., 270, 1 (1990).

22. K. S. Suslick, "Ultrasound: Its Chemical, Physical, and Biological Effects," VCH, New York, N.Y., 1988.

23. K. S. Suslick and M. W. Grinstaff, J. Am. Chem. Soc., 112, 7807 (1990). 